\title{
Time course study of oxyresveratrol inclusion complexes in aqueous solutions
}

$\underline{\text { Irene Conesa }}^{1}$, Silvia Navarro-Orcajada ${ }^{1}$, Adrián Matencio ${ }^{2}$, Iván Muñoz-Sánchez ${ }^{1}$, Lorena Laveda-Cano ${ }^{1}$, Desiré Cano-Yelo ${ }^{1}$, Francisco García-Carmona ${ }^{1}$ and José Manuel LópezNicolás ${ }^{1}$

${ }^{1}$ Departamento de Bioquímica y Biología molecular-A, Facultad de Biología, Universidad de Murcia - Regional Campus of International Excellence "Campus Mare Nostrum”, E-30100 Murcia, Spain

${ }^{2}$ Dipartimento Di Chimica, Università di Torino, via P. Giuria 7, 10125, Torino, Italy

Session: B. Cyclodextrins in pharmaceutics

Abstract: The stilbenes are bioactive molecules with a big amount of health benefits and many possibilities in pharmaceutical industry. Several authors have reported a high number of properties for these compounds, including anticancer, antioxidant, antiinflammatory, antidiabetic, neuroprotective or antimicrobial activities. However, their low aqueous solubility and their ease degradation could lead to low concentration of bioactive compound in the target tissue. For this reason, in the present study, the inclusion complexes of oxyresveratrol with $\alpha-, \beta$-, and $\gamma$ - cyclodextrins are characterized using DSC, TGA, SEM and molecular docking in order to increase the stability of the molecule. All these techniques showed that $\beta$-cyclodextrin $(\beta-C D)$ forms the best complexes.

The stability of oxyresveratrol and oxyresveratrol/ $\beta$-CD complexes in different aqueous solutions was evaluated by measuring ${ }^{\circ} \mathrm{Brix}, \mathrm{pH}$ and UV-Vis spectra. The effect of encapsulation on the solubility and antioxidant activity of oxyresveratrol was also analysed. The results indicated that solutions were stable for at least five weeks, especially when stored in darkness, and that cyclodextrin supplementation leads to a higher concentration and antioxidant capacity of the solubilized bioactive compound than when it is not used. These results and the increase in antioxidant activity could be interesting for the pharmaceutical industry and for drugs enriched in oxyresveratrol.

Keywords: Cyclodextrins, oxyresveratrol, antioxidant activity, encapsulation, stability.

Acknowledgements: This research was funded by the Spanish Ministry of Science and Innovation, project AGL2017-86526-P (MCI/AEI/FEDER, UE) and by the "Programa de Ayudas a Grupos de Excelencia de la Región de Murcia, Fundación Séneca, Agencia de Ciencia y Tecnología de la Región de Murcia (Spain)" (Project 19893/GERM/15).This work is the result of a predoctoral contract for the training of research staff (Silvia Navarro-Orcajada) and also an aid to postdoctoral training and improvement abroad (for Adrián Matencio) both financed by the Consejería de Empleo, Universidades, Empresa y Medio Ambiente of the CARM, through the Fundación Séneca-Agencia de Ciencia y Tecnología de la Región de Murcia. 